\title{
ZPS - Zeitschrift für Psychodrama und Soziometrie
}

Psychodrama und Soziometrie wurden von J. L. Moreno ab 1920 zunächst in Österreich, später in den USA entwickelt. Über ihre Bedeutung als Methoden der Psychotherapie hinaus werden sie in spezialisierter Form in Pädagogik, Organisationsentwicklung, Managementtraining, Theater, Erwachsenenbildung usw. erfolgreich angewendet. Denn wichtige Ziele psychodramatischen und soziometrischen Denkens und Handelns sind die Förderung von Spontaneität und Kreativität sowie die humanere Gestaltung menschlicher Beziehungen auf allen Ebenen des Zusammenlebens.

Die Zeitschrift möchte die vielfältige psychodramatische und soziometrische Theorie und Praxis aus unterschiedlichen Berufsfeldern im deutschsprachigen und internationalen Raum darstellen, neue Konzepte und Verfahren vorstellen und weiter entwickeln, praktisches Handeln vermitteln und zu Forschung anregen.

Jede Ausgabe der Zeitschrift für Psychodrama und Soziometrie ist einem Themenschwerpunkt gewidmet, auf den die Beiträge aus Theorie und Praxis Bezug nehmen.

Anschrift der Redaktion:

ZPS - Zeitschrift für Psychodrama und Soziometrie

Redaktion, Simon-Breu-Straße 30, 97074 Würzburg, Weber-Würzburg@t-online.de

Verzeichnis der Themenhefte:

$1 / 2002$ Wirkfaktor Gruppe*

2 /2002 Trauma, Störung und Ressource*

$1 / 2003$ Rollenwechsel und Rollentausch*

$2 / 2003$ Kreativität wagen

$1 / 2004$ Psychodramatisches Handeln in der Sozialen Arbeit (Hrsg. F. Stimmer)

in Vorbereitung:
$1 / 2005$
$2 / 2005$
Teamentwicklung, Teamberatung
Szenenaufbau
$1 / 2006$
Junge PsychodramatikerInnen
$2 / 2006$
Psychodrama und Gesellschaft
Aggression
(Hrsg. M. Gellert, M. Schacht)
(Hrsg. R. Krüger, K. Weber)
(Hrsg. U. Fangauf, NN.)
(Hrsg. H. Pruckner)
(Hrsg. M. Schacht, K. Weber)

(Die mit * gekennzeichneten Hefte sind leider bereits vergriffen.) 